\title{
Diagnóstico sorológico de infecções por dengue e febre amarela em casos suspeitos no Estado do Pará, Brasil, 1999
}

\author{
Serological diagnosis of dengue and yellow fever infections in \\ suspected cases from Pará State, Brazil, 1999
}

Tais Pinheiro de Araújo ${ }^{2}$, Sueli Guerreiro Rodrigues ${ }^{1}$, Maria Irene Weyl de A. Costa
Pedro Fernando da Costa Vasconcelos ${ }^{1}$ e Amélia P.A. Travassos da Rosa

Resumo De junho a dezembro de 1999, foram coletadas 785 amostras de soro de pacientes com suspeita clínica de dengue e/ou febre amarela. Os pacientes foram atendidos nas unidades de saúde distribuídas pelas seis mesorregiões do Estado do Pará, Brasil. As amostras de soro foram testadas pelo método de inibição da hemaglutinação para detecção de anticorpos para Flavivirus e pelo ensaio imunoenzimático para detecção de imunoglobulina $M$ para dengue e febre amarela. Das amostras coletadas, $563(71,7 \%)$ foram positivas pelo IH, e dentre estas 150 (26,6\%) foram positivas pelo ELISA-IgM. O vírus dengue foi responsável pela maioria das infecções recentes em todas as mesorregiões e os casos de febre amarela detectados neste estudo foram restritos às mesorregiões Marajó e Sudeste.

Palavras-chaves: Flavivirus. Dengue. Febre amarela. Diagnóstico sorológico. Pará. Brasil.

Abstract From June to December 1999, 785 serum samples were obtained from patients clinically suspected of having dengue or yellow fever. The patients were referred by public health centers distributed within the six mesoregions of Pará State, Brazil. Serum samples were tested for Flavivirus antibodies by hemagglutination inhibition test and for dengue and yellow fever viruses by enzyme-linked immunosorbent assay for IgM detection. Of the sera collected, $563(71.7 \%)$ were positive by HI test and out of these $150(26.6 \%)$ were positive by ELISA-IgM. Dengue virus was responsible for most of the recent infections in all regions; yellow fever cases detected in the current study were restricted to the Marajó and Southeast regions.

Key-words: Flavivirus. Dengue fever. Yellow fever. Serological diagnostic. Pará State. Brazil.

Os vírus dengue (DEN) e febre amarela (FA) são membros do gênero Flavivirus, família Flaviviridae ${ }^{18}$. Ambos são importantes arbovírus que causam doença no homem. A introdução do DEN no Estado do Pará ocorreu em 1995, na região sudeste do Estado, nos municípios de Redenção e Rondon do Pará. Os primeiros casos autóctones de dengue no município de Belém, capital do Estado, foram registrados em outubro de 1996, pela detecção de anticorpos IgM para DEN, e em novembro foi isolado e identificado o sorotipo DEN-12. Em outubro de 1997 isolou-se o sorotipo DEN-2, que passou a circular simultaneamente com o $\mathrm{DEN}-{ }^{13}$. Casos humanos de febre amarela no Brasil, sobretudo na Amazônia brasileira, são registrados a cada ano em áreas enzoóticas ou epizoóticas, por transmissão silvestre, ocorrendo esporadicamente ou em surtos ${ }^{16} 17$. A febre amarela silvestre tem sido transmitida pelo mosquito Haemagogus janthinomys, principal vetor no Brasil, e o número de casos tem aumentado nas últimas duas décadas por causa do crescente contacto humano com áreas de floresta ${ }^{316} 17$. A sorologia é essencial no diagnóstico das infecções por Flavivirus e na diferenciação entre infecções primária e secundária. A relação antigênica entre os Flavivirus pode ser demonstrada em ensaios tais como inibição da hemaglutinação $(\mathrm{IH})$ e o ensaio

\footnotetext{
1. Centro Colaborador da Organização Mundial de Saúde em Arboviroses e Laboratório de Referência Nacional de Arbovírus do Instituto Evandro Chagas, Belém, PA, Brasil; 2. Área de Saúde do Centro de Ensino Superior do Pará, Belém, PA, Brasil; 3. Department of Pathology, University of Texas Medical Branch, Galveston, TX, USA.

Fontes de financiamento: IEC/FUNASA/MS e CNPq

Endereço para correspondência: Dra. Tais Pinheiro de Araújo. Av. Almirante Barroso 492, Marco, 66090-000 Belém, PA, Brasil.

Tel: 91 211-4433; Fax: 91 226-5262.

e-mail: pedrovasconcelos@iec.pa.gov.br

Recebido para publicação em 30/5/2001.
} 
imunoenzimático para detecção de IgM (ELISA-IgM), usando anticorpos policlonal e monoclonal ${ }^{20}$. Tradicionalmente, o IH tem sido usado para classificar as respostas imunológicas em primárias (aumento gradual de anticorpos a um título moderado) ou secundárias (aumento rápido a títulos elevados), decorrentes de infecções primárias ou secundárias por Flavivirus, respectivamente, visto que detecta imunoglobulinas totais, principalmente $\lg G$, as quais perduram por um longo período de tempo, constituindo um teste ideal para estudos soro-epidemiológicos. Por outro lado, o teste de ELISA-IgM é muito útil no diagnóstico rápido das infecções correntes e/ou recentes por esses agentes, pois usualmente esta imunoglobulina surge na primeira semana após o início dos sintomas, alcança um pico na segunda semana e perdura por 2 a 3 meses. O presente estudo teve como objetivo esclarecer o diagnóstico de casos suspeitos de dengue e/ou febre amarela, decorrentes de demanda passiva ao Instituto Evandro Chagas (IEC), nas mesorregiões que constituem o Estado do Pará.

\section{MATERIAL E MÉTODOS}

Amostras. Foram incluídas no estudo um total de 785 amostras de soro decorrentes de demanda espontânea ao IEC, no período de junho a dezembro de 1999, para esclarecimento diagnóstico, sendo dengue a principal suspeita clínica. Os pacientes foram atendidos em unidades de saúde distribuídas pelo Estado do Pará, e também no ambulatório da Seção de Arbovírus do IEC, Belém, PA. Todos os pacientes possuíam fichas clínico-epidemiológicas, porém nem sempre devidamente preenchidas.

Áreas estudadas. Diversos municípios das 6 mesorregiões que compõe o Estado do Pará, localizado na região norte do Brasil, com área de $1.253 .164,5 \mathrm{~km}^{2}$ e população de 6.188 .685 habitantes (4.115.774 na área urbana). As amostras apresentaram a seguinte distribuição segundo as mesorregiões: Baixo Amazonas $29(3,7 \%)$, Marajó $9(1,1 \%)$, Metropolitana $257(32,7 \%)$,
Nordeste 126 (16,1\%), Sudeste 347 (44,2\%) e Sudoeste $17(2,2 \%)$.

Testes sorológicos. Os testes sorológicos empregados foram o $\mathrm{IH}$, utilizando a técnica de Clarke e Casals, modificada por Shope ${ }^{14}$, e o ELISAIgM, técnica descrita por Kuno et $\mathrm{a} \Gamma$.

No teste de $\mathrm{IH}$ os soros foram testados para 8 Flavivirus (grupo B dos arbovírus) causadores de doença humana no Brasil, quais sejam: FA (amostras vacinal e silvestre), Ilhéus (ILH), São Luís (SLE), DEN (sorotipos 1 a 4) e Rocio (ROC); cujos antígenos e respectivos soros homólogos foram preparados no IEC, de acordo com procedimentos padrões ${ }^{21014}$. As respostas sorológicas foram classificadas como primária ou secundária para Flavivirus, de acordo com os critérios da Organização Mundial de Saúde $(\mathrm{OMS})^{19}$ e Organização Panamericana de Saúde ${ }^{8}$. No ELISA-IgM os soros foram testados contra DEN e FA.

\section{RESULTADOS}

Amostras e sorologia. Durante os 7 meses do estudo foram testadas 785 amostras de soro pela técnica de $\mathrm{IH}$. Desse total, $563(71,7 \%)$ reagiram com um ou mais Flavivirus e $222(28,3 \%)$ apresentaram sorologia negativa (Tabela 1). Foi possível identificar $89(15,8 \%)$ respostas primárias, com reação monotípica para FA (68), SLE (9), ILH (6) e DEN (6), e 97 (17,2\%) respostas secundárias. As demais amostras ficaram sem interpretação por não se enquadrarem nos critérios de classificação da $\mathrm{OMS}^{19}$. O teste de ELISA-IgM foi aplicado aos 563 soros positivos por IH e detectou-se 144 (96\%) infecções correntes e/ou recentes por DEN e 6 (4\%) por FA.

Mesorregiões. Os soros oriundos das várias mesorregiões apresentaram anticorpos inibidores da hemaglutinação, alcançando percentuais de positividade de $79 \%$ (203) e 88,9\% (8) nas mesorregiões Metropolitana e Marajó, respectivamente (Tabela 1). Pelo ELISA-IgM foram encontrados índices de positividade de $45,8 \%$ (93) para DEN na mesorregião Metropolitana, e 37,5\% (3) para FA no Marajó, e na mesorregião Sudeste o percentual de positividade foi de $10,9 \%$ (27) para DEN e 1,2\% (3) para FA (Tabela 2).

A análise dos resultados de ELISA-IgM, em relação ao total de soro de cada mesorregião, revelou positividade para DEN de 3,4\% (1) no Baixo Amazonas;
$36,2 \%$ (93) na Metropolitana; 18,3\% (23) no Nordeste e 7,8\% (27) no Sudeste. Quanto a FA, 33,3\% (3) dos soros do Marajó apresentaram-se positivos, assim como 0,9\% (3) da mesorregião Sudeste (Figura 1).

O declínio da positividade para DEN observada entre os resultados dos testes de IH e ELISA-IgM alcançou 93,3\% no Baixo Amazonas, 100\% no Marajó, 54,2\% na Metropolitana, $70,1 \%$ no Nordeste e $89,1 \%$ no Sudeste. Enquanto para FA o declínio foi de $62,5 \%$ no Marajó, $98,8 \%$ no Sudeste e de $100 \%$ nas demais mesorregiões (Figura 1).

Positividade para DEN e FA por Sexo e Faixa Etária. A positividade para DEN pelo teste de ELISAIgM foi de $40,3 \%(58 / 144)$ em indivíduos do sexo masculino e de $59,7 \%(86 / 144)$ no sexo feminino (Tabela 3). A análise estatística destes resultados pelo teste de qui-quadrado, com correção de Yates, foi de $x^{2}=10,13$; $p=0,0014$. Dos casos de infecção corrente e/ou recente pelo vírus da FA, 66,7\% (4/6) ocorreram em pessoas do sexo masculino $\left(x^{2}=0,33 ; p=0,5637\right)$.

De acordo com a faixa etária, encontramos que para DEN o grupo 16-30 anos apresentou positividade de $36,1 \%$ (52) do total de 144 pacientes com anticorpos IgM, seguido pelo grupo de $31-45$ anos com $28,5 \%$ (41) (Tabela 3). No que tange a FA, houve uma positividade 
Tabela 1 - Distribuição da positividade do teste de inibição da hemaglutinação para Flavivirus, por mesorregião, Pará, jun/dez 1999.

\begin{tabular}{|c|c|c|c|c|c|}
\hline \multirow[b]{2}{*}{ Mesorregião } & \multicolumn{2}{|c|}{ Positivos } & \multicolumn{2}{|c|}{ Negativos } & \multirow{2}{*}{$\frac{\text { Total }}{\mathrm{n}^{\circ}}$} \\
\hline & $\mathrm{n}^{\circ}$ & $\%$ & $\mathrm{n}^{\circ}$ & $\%$ & \\
\hline Baixo Amazonas & 15 & 51,7 & 14 & 48,3 & 29 \\
\hline Marajó & 8 & 88,9 & 1 & 11,1 & 9 \\
\hline Metropolitana & 203 & 79,0 & 54 & 21,1 & 257 \\
\hline Nordeste & 77 & 61,1 & 49 & 38,9 & 126 \\
\hline Sudeste & 248 & 71,5 & 99 & 28,5 & 347 \\
\hline Sudoeste & 12 & 70,6 & 5 & 29,4 & 17 \\
\hline Total & 563 & 71,7 & 222 & 28,3 & 785 \\
\hline
\end{tabular}

Fonte: Fichas epidemiológicas - Seção de Arbovírus/IEC, junho a dezembro de 1999

Tabela 2 - Distribuição dos resultados do teste de ELISA-IgM para DEN e FA, por mesorregião, Pará, jun/dez 1999.

\begin{tabular}{|c|c|c|c|c|c|c|c|}
\hline \multirow[b]{3}{*}{ Mesorregião } & \multicolumn{4}{|c|}{ Positivos } & & & \multirow{3}{*}{$\begin{array}{c}\text { Total } \\
\mathrm{n}^{\circ}\end{array}$} \\
\hline & \multicolumn{2}{|c|}{ DEN } & \multicolumn{2}{|c|}{ FA } & \multicolumn{2}{|c|}{ Negativos } & \\
\hline & $\mathrm{n}^{\circ}$ & $\%$ & $n^{\circ}$ & $\%$ & $n^{\circ}$ & $\%$ & \\
\hline Baixo Amazonas & 1 & 6,7 & - & - & 14 & 93,3 & 15 \\
\hline Marajó & - & - & 3 & 37,5 & 5 & 62,5 & 8 \\
\hline Metropolitana & 93 & 45,8 & - & - & 110 & 54,2 & 203 \\
\hline Nordeste & 23 & 29,9 & - & - & 54 & 70,1 & 77 \\
\hline Sudeste & 27 & 10,9 & 3 & 1,2 & 218 & 87,9 & 248 \\
\hline Sudoeste & - & - & - & - & 12 & 100,0 & 12 \\
\hline Total & 144 & 25,6 & 6 & 1,1 & 413 & 73,4 & 563 \\
\hline
\end{tabular}

Elisa-IgM: ensaio imunoenzimático para detecção de IgM. DEN: vírus dengue. FA: febre amarela.

Fonte: Fichas epidemiológicas - Seção de Arbovírus/IEC, junho a dezembro de 1999

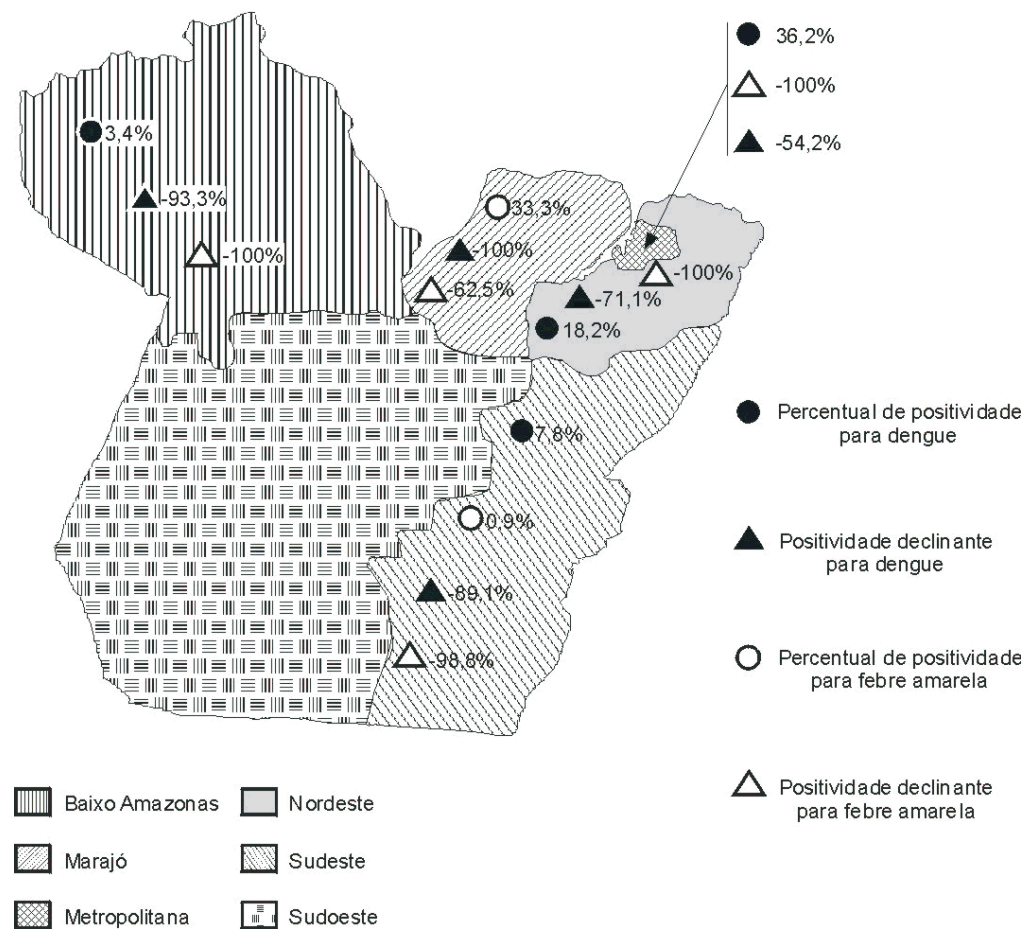

Figura 1 - Distribuição dos percentuais de positividade, em relação ao total de soros, e declínio da positividade observada entre os resultados de IH e ELISAlgM, por mesorregião, Pará, junho a dezembro 1999.

Fonte: Fichas epidemiológicas - Seção de Arbovírus/IEC, junho a dezembro de 1999. 
Tabela 3 - Distribuição das infecções por dengue por sexo e faixa etária, Pará, jun/dez

\begin{tabular}{|c|c|c|c|c|c|c|}
\hline \multirow[t]{2}{*}{ Faixa etária (anos) } & \multicolumn{2}{|c|}{ Masculino } & \multicolumn{2}{|c|}{ Feminino } & \multicolumn{2}{|c|}{ Total } \\
\hline & $\mathrm{n}^{\circ}$ & $\%$ & $\mathrm{n}^{\circ}$ & $\%$ & $\mathrm{n}^{\circ}$ & $\%$ \\
\hline$\leq 15$ & 10 & 66,7 & 5 & 33,3 & 15 & 10,4 \\
\hline $16-30$ & 16 & 30,8 & 36 & 69,2 & 52 & 36,1 \\
\hline $31-45$ & 19 & 46,3 & 22 & 53,7 & 41 & 28,4 \\
\hline $46-60$ & 5 & 22,7 & 17 & 77,3 & 22 & 15,3 \\
\hline$\geq 61$ & 3 & 50,0 & 3 & 50,0 & 6 & 4,2 \\
\hline Ignorada & 5 & 62,5 & 3 & 37,5 & 8 & 5,6 \\
\hline Total & 58 & 40,3 & 86 & 59,7 & 144 & 100 \\
\hline
\end{tabular}

Fonte: Fichas epidemiológicas - Seção de Arbovírus/IEC, junho a dezembro de 1999.

de $66,6 \%$ (4) na faixa etária de 16-30 anos e 16,7\% (1) em cada das faixas etárias de 31-45 e $\leq 15$ anos.

Presença de anticorpos $x$ tempo de doença. As fichas epidemiológicas de $46,5 \%$ dos pacientes não continham informação de tempo de doença, sendo classificado como ignorado (Tabela 4). Dentre os pacientes com anticorpos inibidores da hemaglutinação 108 $(19,2 \%)$ e $207(36,8 \%)$ estavam com $\leq 5$ e $\geq 6$ dias de doença, respectivamente. Detectou-se anticorpos IgM anti-DEN em $90(62,4 \%)$ e anti-FA em 2 (33,3\%) dos pacientes com $\geq 6$ dias de doença. Anticorpos IgM antiDEN também foram detectados em 27 (18,8\%) dos pacientes com $\leq 5$ dias de doença (Tabela 4).

Tabela 4 - Distribuição dos resultados pelo teste de IH e ELISA-IgM quanto ao tempo de doença, Pará, jun/dez 1999.

\begin{tabular}{|c|c|c|c|c|c|c|c|c|c|c|}
\hline \multirow{3}{*}{$\begin{array}{l}\text { Tempo de } \\
\text { doença (dias) }\end{array}$} & & & \multicolumn{4}{|c|}{$\mathrm{IH}$} & \multicolumn{4}{|c|}{ ELISA-IgM } \\
\hline & \multicolumn{2}{|c|}{ Total } & \multicolumn{2}{|c|}{ negativos } & \multicolumn{2}{|c|}{ Flavivirus } & \multicolumn{2}{|c|}{ DEN } & \multicolumn{2}{|c|}{ FA } \\
\hline & $n$ & $\%$ & $\mathrm{n}$ & $\%$ & $\mathrm{n}$ & $\%$ & $\mathrm{n}$ & $\%$ & $\mathrm{n}$ & $\%$ \\
\hline$\leq 5$ & 151 & 19,2 & 43 & 19,4 & 108 & 19,2 & 27 & 18,8 & - & - \\
\hline$\geq 6$ & 269 & 34,3 & 62 & 27,9 & 207 & 36,8 & 90 & 62,4 & 2 & 33,3 \\
\hline Ignorado & 365 & 46,5 & 117 & 52,7 & 248 & 44,0 & 27 & 18,8 & 4 & 66,7 \\
\hline Total & 785 & 100,0 & 222 & 100,0 & 563 & 100,0 & 144 & 100,0 & 6 & 100,0 \\
\hline
\end{tabular}

IH: inibição da hemoglatinação. ELISA-IgM: Ensaio imunoenzimático para detecção de IgM. DEN: vírus dengue. FA: febre amarela.

Fonte: Fichas epidemiológicas - Seção de Arbovírus/IEC, junho a dezembro de 1999.

\section{DISCUSSÃO}

A positividade de anticorpos inibidores da hemaglutinação para Flavivirus foi superior a 50\% em todas as mesorregiões do Pará (Tabela 1), provavelmente decorrente da ampla circulação do vírus DEN e, também, como conseqüência de resposta vacinal contra o vírus FA. O Estado do Pará pertence a área endêmica de febre amarela silvestre, onde a vacinação anti-amarílica é amplamente administrada. Além disso, a partir de 1998, esta cobertura vacinal foi intensificada em todo o território nacional devido ao risco de reurbanização da FA frente ao aumento da atividade viral em zona endêmica, dispersão e densidade do vetor urbano (Aedes aegypti), bem como a crescente mobilidade da população humana ${ }^{15}$. Ademais, a Amazônia brasileira possui condições climáticas favoráveis a par de elevada densidade e diversidade de artrópodes hematófagos e animais silvestres, que constituem os elementos básicos para a manutenção dos arbovírus ${ }^{9}$. A detecção de reações monotípicas por $\mathrm{IH}$, nas diversas mesorregiões, para SLE (9) e ILH (6), Flavivirus encefalitogênicos para o homem, sugere a circulação desses vírus.

Foi confirmado um maior número de infecções pelo DEN nas mesorregiões Metropolitana, Nordeste e
Sudeste (Tabela 2), que correspondem a zona mais populosa e desenvolvida do Estado. Nestas mesorregiões, provavelmente, a dispersão do vírus foi favorecida pela maior mobilidade de suas populações, bem como pelo desenvolvimento e proliferação do mosquito Aedes aegypti decorrente de fatores tais como, reurbanização sem infraestrutura básica de saneamento, coleta inadequada do lixo, desmatamento desordenado ${ }^{12}$. O DEN-1 foi introduzido no Estado pela mesorregião Sudeste em 1995 e cerca de 1 ano depois causava epidemia na capital do Estado, na mesorregião Metropolitana.

Um estudo realizado para avaliar os índices de sensibilidade e especificidade, bem como as taxas de falso-negativos e falso-positivos do ELISA-IgM para DEN em relação ao $\mathrm{IH}^{1}$, demonstrou a correlação de resultados entre ambas as técnicas. Entretanto, na presente investigação sorológica, apesar de muitas amostras procederem de quadros clínicos típicos de dengue, apenas 144 (25,6\%) dos pacientes com anticorpos para Flavivirus foram confirmadas como infecção recente para este vírus (Tabela 2).

A detecção de anticorpos IgM pelo ELISA-IgM para DEN quando comparados com a positividade para 
Flavivirus obtida no $\mathrm{IH}$, revelou um declínio de positividade maior que o esperado (Figura 1), já que esta foi a principal suspeita clínica que motivou a demanda de amostras. O acentuado declínio pode ter sido decorrente de resultados falso-negativos no ELISA-IgM, devido a coleta de soro até o $5^{\circ}$ dia após o início dos sintomas, bem como, por conta de infecção secundária por Flavivirus. Em ambos os casos, pode ocorrer produção de anticorpos IgM a níveis não detectáveis ${ }^{7}$. Além disso, o quadro clínico de alguns pacientes poderia não corresponder a infecção pelo DEN, e, neste caso, os anticorpos inibidores da hemaglutinação detectados seriam decorrentes de infecções anteriores por algum Flavivirus, ou vacinação antiamarílica.

A positividade para DEN (62,4\%) entre os pacientes com $\geq 6$ dias de doença foi bem maior do que entre aqueles com $\leq 5$ dias de doença $(18,8 \%)$, sendo esta diferença estatísticamente significativa, com $x^{2}=65,71 ; p<0,0001$ (Tabela 4). O percentual de positividade de $18,8 \%$ observado entre os pacientes com suspeita de DEN e tempo de doença ignorado sugere coleta do soro em momento inadequado para pesquisa de anticorpos IgM (Tabela 4).

A literatura relata um maior número de infecções por DEN em pessoas do sexo feminino, possivelmente devido a um maior tempo de permanência nas residências, e conseqüentemente maior exposição ao vetor ${ }^{4}$. A análise estatística dos resultados obtidos em nosso estudo revelou diferença significativa de infecção entre os sexos $\left(x^{2}=10,13 ; p=0,0014\right)$ mostrando que o risco de transmissão do DEN foi maior nas mulheres. Os resultados mostraram também que todas as faixas etárias foram acometidas, sendo que o grupo mais vulnerável foi o de 16-30 anos, seguido pelo grupo de 31-45 anos (Tabela 3), portanto aqueles com maior atividade produtiva.

Os casos de FA detectados neste estudo ocorreram em indivíduos não imunizados contra FA e residentes em zona rural, nas mesorregiões Marajó e Sudeste, áreas endêmicas do vírus, que freqüentemente registram casos esporádicos de FA silvestre. O elevado declínio de positividade entre o IH e o ELISA-IgM para FA reflete a atuação da vigilância epidemiológica para manter a FA urbana erradicada e a FA silvestre sob controle. Na última década foram registrados cerca de 73 casos de FA silvestre no Estado do Pará6, nenhum deles nas mesorregiões Nordeste e Metropolitana. $O$ pequeno percentual de amostras decorrente de pacientes com suspeita de FA foram oriundas, principalmente, das mesorregiões Marajó e Sudeste.

Dos seis casos de FA, quatro ocorreram em pessoas do sexo masculino, o que está de acordo com a literatura ${ }^{5}$. A análise dos casos de FA, segundo a faixa etária dos pacientes (dados não mostrados), identificou um caso entre indivíduos $\leq 15$ anos na mesorregião do Marajó. Este fato pode indicar que as crianças desempenham um papel importante no auxílio aos pais no trabalho, e se infectam quando penetram no nicho de transmissão do vírus, fato já evidenciado em outros estudos ${ }^{16}{ }^{17}$. Contudo, pode também significar que na região da Ilha do Marajó, as pessoas foram mais infectadas no domicílio e/ou peridomicílio, já que o deslocamento nessa região é dificultado pela presença de áreas alagadiças e pela limitação do número de barcos e canoas, os principais meios de transporte usados pelas pessoas. Vale ressaltar que em 1984 observou-se um surto no Baixo Amazonas onde registraram-se 31 casos, sendo 7 $(22,6 \%)$ em mulheres e $5(16,1 \%)$ em crianças, e nesta oportunidade identificou-se um novo vetor de FA silvestre no Brasil, Haemagogus albomaculatus ${ }^{11}$. Este mosquito tem sido incriminado na transmissão humana peridoméstica na Amazônia Central, visto que apresenta maior autonomia de vôo, podendo chegar ao domicílio e peridomicílio para picar ${ }^{317}$.

Os achados sorológicos sugerem ainda que afora DEN e FA, outros Flavivirus tais como SLE e ILH, circulam na região e podem ser responsáveis por quadros febris agudos sem diagnóstico definido.

\section{AGRADECIMENTOS}

A todos os funcionários da Seção de Arbovírus do Instituto Evandro Chagas/FUNASA/MS que contribuíram para o desenvolvimento deste estudo e ao Dr. Robert Tesh da University of Texas Medical Branch pela revisão do inglês.

\section{REFERÊNCIAS BIBLIOGRÁFICAS}

1. Araújo TP, Nunes MRT, Cerqueira DIS, Rodrigues SG, Travassos da Rosa APA. Evaluation of the MAC-ELISA technique for the serological diagnosis of dengue virus infection among patients from Belém, Pará, 1998. Virus Reviews and Research. Sociedade Brasileira de Virologia 4: 122, 1999.

2. Beaty BJ, Calisher $\mathrm{CH}$, Shope RE. Arboviruses. In: Lennette EH, Lennette DA, Lennette ET (eds) Diagnostic Procedures for Viral, Rickettsial and Chlamydial Infections, $7^{\text {th }}$ edition. American Public Health Association, Washington, p.189-212, 1995.

3. Dégallier N, Travassos da Rosa APA, Hervé JP, Travassos da Rosa JFS, Vasconcelos PFC, Silva CJM, Barros VLRS, Dias LB, Travassos da Rosa ES, Rodrigues SG. A comparative study of yellow fever in Africa and South America. Journal of the Brazilian Association for the Advancement of Science 44:143-151, 1992.

4. Fundação Nacional de Saúde. Manual de Dengue - Vigilância Epidemiológica e Atenção ao Doente. Ministério da Saúde. Brasília, 1995.

5. Fundação Nacional de Saúde. Manual de Vigilância Epidemiológica de Febre Amarela. Ministério da Saúde, Brasília, 1999.

6. Fundação Nacional de Saúde. Relatório Técnico. CENEPI/ CGVEPI/COVEV, Ministério da Saúde. 2001.

7. Kuno G, Gomez I, Gubler, DJ. Detecting artificial antidengue IgM immune complexes using an enzyme-linked immunosorbent 
assay. The American Journal of Tropical Medicine and Hygiene 36:153-159, 1987.

8. Pan American Health Organization. Dengue and dengue hemorrhagic fever in the Americas: Guidelines for prevention and control, Washington, 1994.

9. Pinheiro FP, Travassos da Rosa APA, Vasconcelos PFC. Arboviroses. In: Veronesi R, Focaccia R (eds) Tratado de Infectologia, Atheneu, São Paulo, p.169-180, 1996.

10. Rosa APAT, Travassos da Rosa ES, Travassos da Rosa JFS, Dégallier N, Vasconcelos PFC, Rodrigues SG. Os Arbovírus no Brasil: Generalidades, Métodos e Técnicas de Estudo. Documento Técnico. $\mathrm{n}^{\circ}$ 2. Instituto Evandro Chagas/Fundação Nacional de Saúde, Belém, 1994.

11. Rosa APAT, Vasconcelos PFC, Hervé JP, Travassos da Rosa JFS. Febre amarela silvestre no Estado do Pará - Brasil, 1984. Boletim Epidemiológico Fundação SESP 16: 97-104, 1984.

12. Rosa APAT, Vasconcelos PFC, Pinheiro FP, Travassos da Rosa JFS, Rodrigues, SG. Dengue. In: Leão RNQ (ed) Doenças Infecciosas e Parasitárias - Enfoque Amazônico, Cejup/UEPA/ Instituto Evandro Chagas, Belém, p. 227-241, 1997.

13. Rosa APAT, Vasconcelos PFC, Travassos da Rosa ES, Rodrigues SG, Mondet B, Cruz ACR, Sousa MR, Travassos da Rosa JFS. Dengue epidemic in Belem, Para, Brazil, 1996-97. Emerging Infectious Diseases 6:298-301, 2000.

14. Shope RE. The use of a microhemagglutination-inhibition test to follow antibody response after arthropod-borne virus infection in a community of forest animals. Anais de Microbiologia 11 (parte A):167-171, 1963
15. Vasconcelos PFC, Luna EJ, Galler R, Silva LJ, Coimbra TL, Barros VLRS, Monath TP, Rodrigues SG, Laval C, Costa ZG, Vilela MFG, Santos CLS, Papaiordanou CMO, Alves VAF, Andrade LD, Sato HK, Rosa EST, Froguas GB, Lacava E, Almeida LMR, Cruz ACR, Rocco IM, Santos RTM, Oliva OFP,Papaiordanou CM. Serious adverse events associated with yellow fever 17DD vaccine in Brazil: a report of two cases. The Lancet 358:91-97, 2001.

16. Vasconcelos PFC, Rodrigues SG, Dégallier N, Moraes MAP, Travassos da Rosa JFS, Travassos da Rosa ES, Mondet B, Barros VLRS, Travassos da Rosa APA. An epidemic of sylvatic yellow fever in the southeast region of Maranhao State, Brazil, 19931994: epidemiologic and entomologic findings. The American Journal of Tropical Medicine and Hygiene 57:132-137, 1997.

17. Vasconcelos PFC, Travassos da Rosa APA, Pinheiro FP, Dégallier N, Travassos da Rosa JFS. Febre amarela. In: Leão RNQ (ed) Doenças Infecciosas e Parasitárias - Enfoque Amazônico, Cejup/ UEPA/Instituto Evandro Chagas, Belém, p. .265-284, 1997.

18. Westaway EG, Brinton MA, Gaidamovich SYA, Horzinek MC, Igarashi A, Kääriäinnen L, Lvov DK, Porterfield JS, Russel PK, Trent DW. Flaviviridae. Intervirology 24:183-192, 1985.

19. World Health Organization. Dengue haemorrhagic fever: diagnosis, treatment and control, $2^{\text {nd }}$ edition, Geneva, 1997.

20. van Regenmortel MHV, Fauquet CM, Bishop DHL, Carstens EB, Estes MK, Lemon SM, Maniloff J, Mayo MA, McGeoch DJ, Pringle CR, Wickner RB. Virus Taxonomy. The Classification and Nomenclature of Viruses. The Seventh Report of the International Committee on Taxonomy of Viruses (book). Virus Taxonomy, VII report of the ICTV, Academic Press, San Diego, 2000. 